Check for updates

Cite this: Mater. Adv., 2021,

2, 1072

\title{
Fluorinated porous silicon as sensor material for environmentally toxic gases: a first-principles study
}

\author{
José Eduardo Santana, ${ }^{a}$ Francisco de Santiago, (D) ${ }^{a}$ Álvaro Miranda, (D) *a \\ Luis Antonio Pérez, ${ }^{b}$ Fernando Salazar, ${ }^{a}$ Alejandro Trejo ${ }^{a}$ and Miguel Cruz-Irisson ${ }^{a}$ \\ By using Density Functional Theory, the effect of adsorbed gas molecules on the electronic properties \\ of fluorine passivated porous silicon ( $\mathrm{pSi}$ ) is investigated. A silicon nanopore is created by removing \\ columns of atoms along the [001] crystallographic axis from a supercell of the bulk $\mathrm{Si}$ crystal. The $\mathrm{Si}$ \\ dangling bonds of the generated pore are saturated with fluorine atoms except for the sites where gas \\ molecules of $\mathrm{NO}, \mathrm{NO}_{2}$ and $\mathrm{SO}_{2}$ are adsorbed. The adsorption energies, electronic densities of states and \\ band structures of the different complexes formed by the nanopore and the adsorbed molecules are \\ calculated and compared with previously reported results obtained for hydrogen-passivated pSi. The \\ energy band gaps of the pSi-molecule complexes depend on the adsorbed species, opening \\ the possibility of gas molecule recognition. The molecule adsorption energy is stronger for $\mathrm{NO}_{2}$. The \\ understanding of molecule adsorption on silicon nanopores could lead to the development of novel \\ sensing devices of environmentally hazardous gases.
}

Received 13th November 2020 Accepted 29th December 2020

DOI: $10.1039 / \mathrm{d} 0 \mathrm{ma} 00884 \mathrm{~b}$

rsc.li/materials-advances
(HF) of crystalline silicon substrates, ${ }^{17-19}$ porous silicon has generated great interest in the scientific community. Fast preparation, tuneable optical and electronic properties, large inner surface area, surface reactivity, good stability and full compatibility with modern electronic technology are some of the advantageous properties of pSi. In particular, a large surface area is required for a good molecule adsorption and pSi etched on p-type $\operatorname{Si}(100)$ provides a surface area of $\sim 200 \mathrm{~m}^{2} \mathrm{~cm}^{-3}$ and a site density of $6.8 \times 10^{14}$ sites per $\mathrm{cm}^{2} \cdot{ }^{20-22}$ Additionally, it has been proven that $\mathrm{pSi}$ can operate in a voltage range of 1-10 $\mathrm{mV}$ with a sensitivity range of $10-100 \mathrm{ppm}^{23}$ This allows the use of $\mathrm{pSi}$ in sensing electronic devices, ranging from the detection of several chemical agents to the identification of DNA chains and proteins. $^{15,24-26}$ Moreover, several experimental studies have been done to prove that pSi, grown in [100] Si wafers, can be an active gas sensing device. ${ }^{5,27-30}$

In a similar way, surface chemistry has been proved to modify the electronic structures and optical behaviour of semiconductors. ${ }^{31}$ Hydrogen passivation causes a transition from metallic to semiconducting behaviour in Si nanowires ${ }^{32,33}$ and band gap changes in another Si nanostructures. ${ }^{34-37}$ Previous studies show that single-bonded atoms on Si surfaces, for example, $\mathrm{Cl}$ and $\mathrm{F}$, may lightly diminish the energy bandgap. ${ }^{38-40}$ The same occurs with double-bonded atoms, such as $\mathrm{N}$ and $\mathrm{O}$, but the bandgap decrement is larger in comparison with singlebonded atoms. ${ }^{41,42}$ However, it has been experimentally observed that the H-passivated pSi surface is rapidly oxidized in
${ }^{a}$ Instituto Politécnico Nacional, ESIME-Culhuacán, Av. Santa Ana 1000, 04430, Ciudad de México, Mexico.E-mail: amirandad.ipn@gmail.com

${ }^{b}$ Instituto de Física, Universidad Nacional Autónoma de México, C.P. 04510, Ciudad de México, Mexico 
air, ${ }^{43,44}$ which leads the surface to irreversible modification and, consequently, a decrease in the sensitivity of this material to molecules such as $\mathrm{NO}_{2}{ }^{45}$ Therefore, the surface fluorination of $\mathrm{pSi}$ is a key ingredient in this work, considering that the presence of fluorine atoms on the Si surface could inhibit the formation of silicon oxide ${ }^{46}$ and make the dissociation of adsorbed oxygencontaining molecules less likely. Even though the interactions between silicon surfaces and fluorine-containing molecules are complicated, the fluorination process of Si surfaces has been extensively studied. $\mathrm{Wu}$ and Carter report that $\mathrm{F}$ atoms only saturate the Si-dandling bonds. ${ }^{47}$ Also, Hoshino and Nishioka indicate that the development of F-passivated silicon surfaces is possible after a chemical reaction of hydrogen fluoride (HF) with H-passivated silicon surfaces. ${ }^{48,49}$ Otherwise, the fluorination of $\mathrm{H}$-passivated silicon surfaces has been recently studied using different F-containing molecules. ${ }^{50}$ Fluorine-passivation is a feasible and advantageous way of tuning the electronic properties of pSi.

In a previous work, the adsorption of hazardous gas molecules, such as $\mathrm{CO}, \mathrm{NO}, \mathrm{NO}_{2}$ and $\mathrm{SO}_{2}$, on pSi passivated with hydrogen $(\mathrm{pSi}+\mathrm{H})$ was studied. ${ }^{15}$ Concerning to the fluorinepassivated $\mathrm{pSi}(\mathrm{pSi}+\mathrm{F})$, there is not, to the best of our knowledge, a theoretical investigation of the adsorption properties of this passivation. For this reason, in this work, we study the effects of fluorine surface passivation of $\mathrm{pSi}$ on the adsorption of hazardous gas molecules such as $\mathrm{NO}_{2}, \mathrm{SO}_{2}$, and $\mathrm{NO}$, and compare these results with those previously reported for $\mathrm{pSi}+\mathrm{H}$. To this end, the electronic band structures and energetic stabilities of the formed molecule-nanopore complexes, together with the molecule adsorption energies, charge excesses and desorption times, are analyzed. The results obtained are related to the potential application of $\mathrm{pSi}+\mathrm{F}$ as chemical sensors.

\section{Computational details and model}

Density-Functional-Theory (DFT) $)^{51,52}$ calculations were carried out using the Perdew-Burke-Ernzerhof (PBE) exchange-correlation functional within the generalized-gradient approximation (GGA), ${ }^{53}$ with double- $\zeta$ polarized basis sets ${ }^{54,55}$ and normconserving Troullier-Martins pseudopotentials ${ }^{56}$ in their fully non-local form ${ }^{57}$ as implemented in the SIESTA code. ${ }^{58}$ For numerical integrations, a real-space grid defined by an energy cutoff of 160 Ry was considered. Geometry optimization was done through a conjugate gradient algorithm until the HellmannFeynman forces were less than $0.04 \mathrm{eV}^{-1}$, with an average maximum value of $0.037 \mathrm{eV} \AA^{-1}$. The average energy change per atom was $2.79 \times 10^{-6} \mathrm{eV}$, the maximum atomic displacement allowed in the optimization process was $0.105 \AA$, and the maximum and minimum obtained values for the atomic displacements were $2.52 \times 10^{-3} \AA$ and $9.56 \times 10^{-5} \AA$, for $\mathrm{pSi}+\mathrm{H}$, respectively. Likewise, for $\mathrm{pSi}+\mathrm{F}$, the corresponding values were $2.08 \times 10^{-3} \AA$ and $4.37 \times 10^{-5} \AA$.

Following the Monkhorst-Pack scheme, the first Brillouin zone of the studied systems was sampled with a grid of $2 \times 2 \times 2$ $k$-points. ${ }^{59}$ The Voronoi electronic population analysis was used to obtain the electronic charge transfer between gas molecules and the passivated $\mathrm{pSi}^{60}$

A porous silicon supercell with $38.28 \%$ porosity is obtained by removing a column of atoms along the [001] direction of a bulk Si supercell, leading to $\{110\}$ internal pore surfaces. The bulk crystalline Si supercell is formed by $4 \times 4 \times 4$ cubic unit cells ( 8 atoms per unit cell). The resulting porous supercell, with periodic boundary conditions along the three spatial directions, has cell parameters $a=b=c=22.09 \AA$.

The dangling bonds of the exposed surfaces were saturated with fluorine atoms before the structural optimization was carried out, to prevent their reconstruction.

After the structural optimization of the fully passivated nanopores, one passivating atom was detached from a $\{110\}$ facet to generate a Si dangling bond, and then this structure was structurally relaxed again. Fig. 1 shows the optimized passivated $\mathrm{pSi}$ structures together with the equilibrium configurations of the adsorbed molecules ( $\mathrm{NO}, \mathrm{NO}_{2}$ and $\mathrm{SO}_{2}$ ). The dangling bond

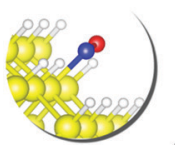

a)

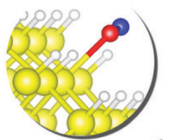

b)
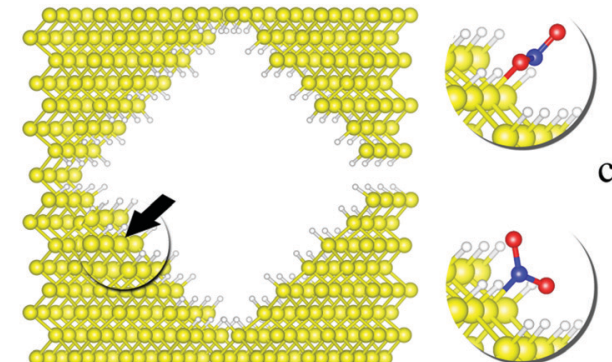

c)

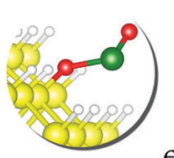

e)

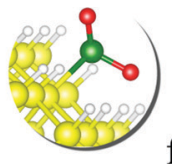

f)

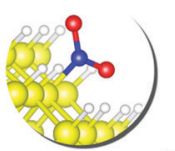

d)

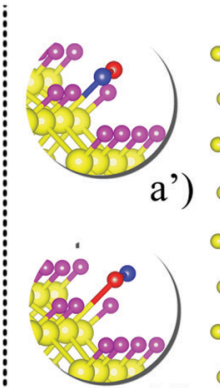

b')
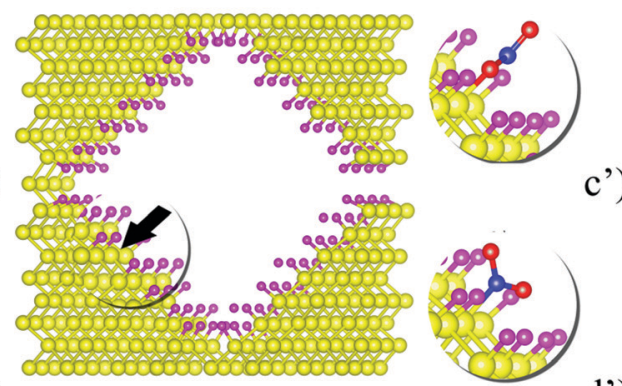

d')

Fig. 1 Optimized structures of pSi with $\mathrm{H}$ - (left panel) and F-passivation (right panel); together with the equilibrium configurations of (a and $\left.\mathrm{a}^{\prime}\right) \mathrm{NO}(\mathrm{N})$, ( $b$ and $\left.b^{\prime}\right) \mathrm{NO}(\mathrm{O})$, (c and $\left.c^{\prime}\right) \mathrm{NO}_{2}(\mathrm{O})$, ( $\left(\mathrm{d}\right.$ and $\left.\mathrm{d}^{\prime}\right) \mathrm{NO}_{2}(\mathrm{~N})$, (e and $\left.\mathrm{e}^{\prime}\right) \mathrm{SO}_{2}(\mathrm{~S})$, ( $f$ and $\mathrm{f}^{\prime}$ ) and $\mathrm{SO}_{2}(\mathrm{O})$ adsorbed molecules. The symbol in parenthesis indicates which atom of the adsorbed molecule is bonded to the pSi dangling bond. Yellow, white, magenta, green, blue and red spheres represent $\mathrm{Si}, \mathrm{H}, \mathrm{F}, \mathrm{S}, \mathrm{N}$ and $\mathrm{O}$ atoms, respectively. 
sites (black arrows on Fig. 1) were used as adsorption sites for the molecules. For comparison purposes the same process was performed with $\mathrm{H}$ passivation, considering, as stated before, that the potential adsorption of these molecules would eventually lead to an oxidation of the pSi surface and a loss of sensing capabilities.

\section{Results and discussion}

In this section, we analyse the electronic band structure of F-passivated pSi, the HOMO-LUMO gap, the charge transfer from the molecule to the pSi, the molecule adsorption energy, the desorption time and the bond distance between the molecule and pSi.

\section{Hydrogen- and fluorine-passivated porous silicon}

Fig. 2 and 3 show the charge density difference (CCD), the electronic band structure and PDOS of the structurally relaxed $\mathrm{pSi}+\mathrm{H}$ and $\mathrm{pSi}+\mathrm{F}$, respectively.

The orange (gray) isosurfaces in Fig. $2 \mathrm{~b}$ and $3 \mathrm{~b}$ illustrate the gain (loss) of electronic charge in comparison with the groundstate charge density of the isolated atoms, for the pSi systems. They are given by:

$$
\delta \rho(\vec{r})=\rho_{\mathrm{X}}(\vec{r})-\rho_{\mathrm{M}}(\vec{r}),
$$

where $\rho_{\mathrm{X}}(r)$ is the charge density of the pSi with $\mathrm{X}$ passivation obtained from the DFT self-consistent calculations, while $\rho_{\mathrm{M}}(r)$ is the electronic charge density resulting from the sum of the ground-state charge densities of each atom. Both terms in eqn (1) are calculated with each atom of the system situated at the position of the optimized structure.

The Voronoi charge transfer from passivating atoms to $\mathrm{Si}$ atoms is markedly different between the $\mathrm{F}$ and $\mathrm{H}$ cases, having

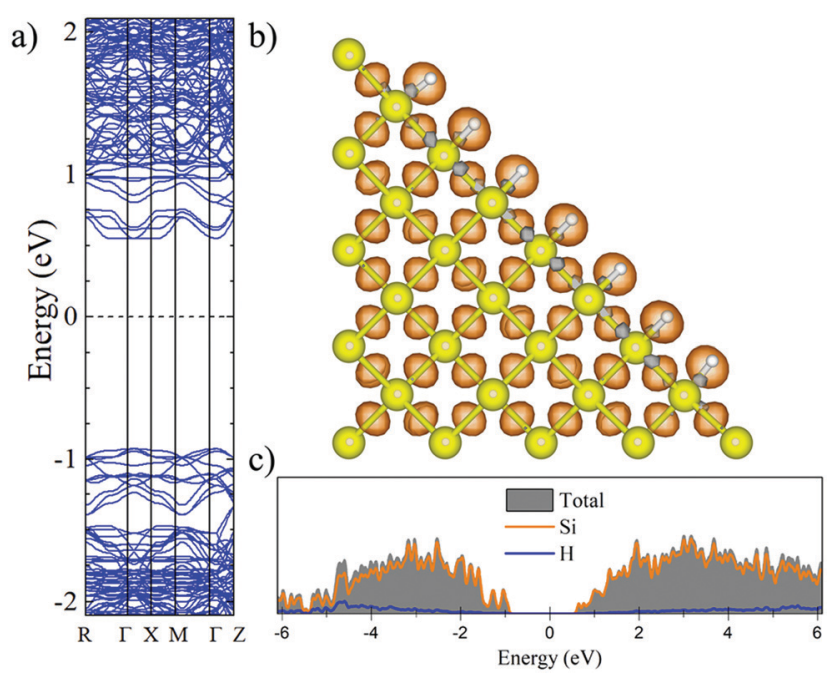

Fig. 2 Electronic band structure (a), charge density difference (b) and PDOS (c) of the totally hydrogenated pSi after geometrical optimization. Orange (gray) isosurfaces denote gain (loss) of electronic charge in comparison with the ground-state charge density of the isolated atoms, for $\mathrm{H}$-passivated pSi.

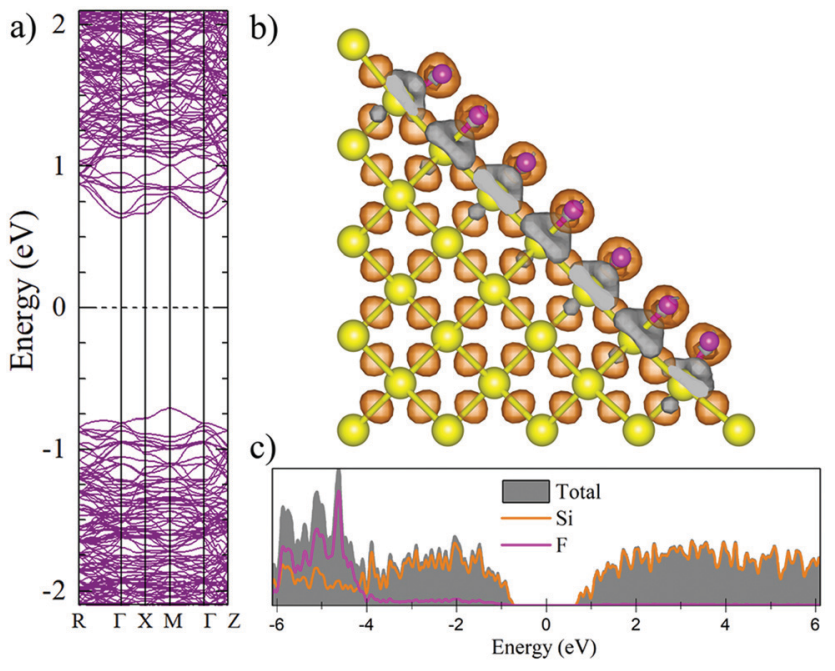

Fig. 3 Electronic band structure (a), charge density difference (b) and partial electronic densities of states (c) of the fluorinated pSi after geometrical optimization. Orange (gray) isosurfaces denote gain (loss) of electronic charge in comparison with the ground-state charge density of the isolated atoms, for F-passivated pSi.

values of $-0.1126|e|$ and $-0.0435|e|$, respectively. In case of F-passivation (Fig. 3b), we find a prominent redistribution of charge around the $\mathrm{F}$ atoms and on the Si surface (gray isosurface) in comparison with the H-passivated system (Fig. 2b). Also, we can see a decrement in the bandgap energy from $1.48 \mathrm{eV}$ for $\mathrm{H}$-passivation to $1.34 \mathrm{eV}$ for F-passivation (Fig. 2a and 3a). This difference can be better understood with the partial density of species (PDOS) representation shown in Fig. 2c and 3c. The bonding energy between a surface $\mathrm{Si}$ and an $\mathrm{H}$ atom is $4.6 \mathrm{eV}$, indicating that the $\mathrm{Si}-\mathrm{H}$ bonds are stable. ${ }^{61}$ The difference in electronegativities between $\mathrm{H}$ (2.2) and $\mathrm{Si}$ (1.9), indicates that this is a polar covalent bond, which is corroborated by both the charge excess on the $\mathrm{H}$ atoms and the charge density isosurfaces, where the higher charge density values are predominantly found close to the $\mathrm{H}$ atoms. There are small grey lobes around the $\mathrm{Si}$ surface atoms, which indicates that part of its charge gets transferred to the $\mathrm{H}$ as expected. Comparatively the $\mathrm{F}$ has similar characteristics, however, due to the much higher electronegativity of $\mathrm{F}$, the bond became almost ionic in nature, and the surface silicon atoms undergo a greater charge loss, thus reflected in a narrower band gap due to the increase of available states of the surface Si atoms.

\section{Adsorption and desorption properties}

Fig. 4 shows the adsorption energy $\left(E_{\mathrm{A}}\right)$ and the bond distance $(\beta)$ between the molecules and the passivated pSi. For each molecule, we consider that bonding with pSi may be possible through each of their constituent elements, for example, in the case of $\mathrm{NO}_{2}$, we consider the cases in which $\mathrm{N}-\mathrm{Si}$ or O-Si bonds may be created. The adsorption energy $\left(E_{\mathrm{A}}\right)$ of a molecule adsorbed on the pSi is given by: ${ }^{14}$

$$
E_{\mathrm{A}}=E_{\mathrm{Mol}}+E_{\mathrm{X}+\mathrm{DB}}-E_{\mathrm{X}+\mathrm{DB}+\mathrm{Mol}}+E_{\mathrm{BSSE}},
$$




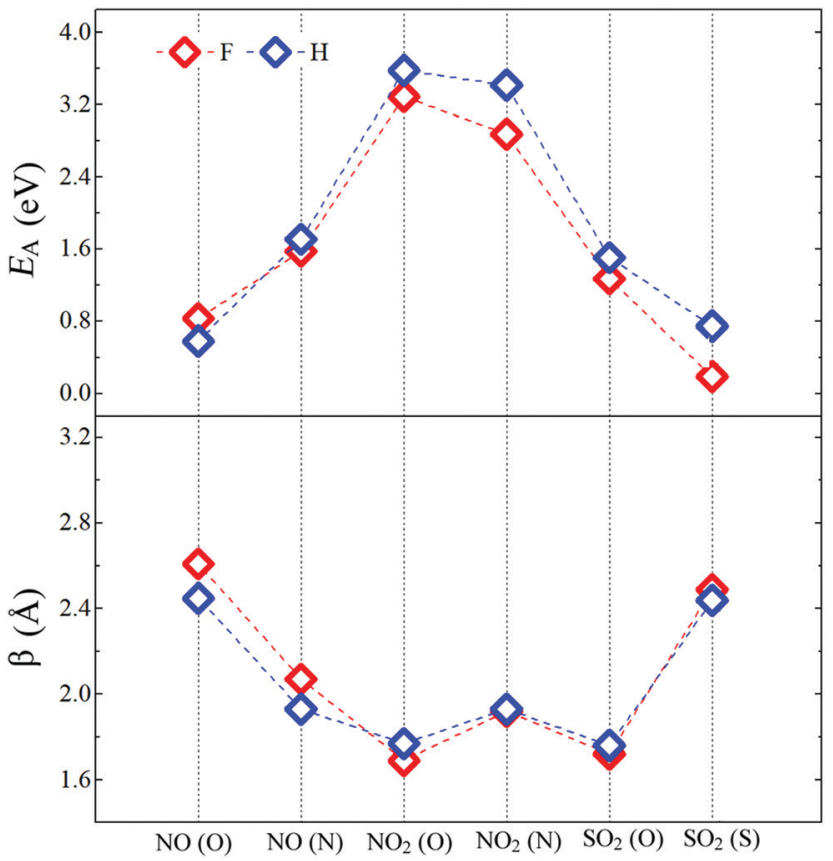

Fig. 4 Adsorption energies $\left(E_{\mathrm{A}}\right)$ and bond distances $(\beta)$ between molecules and $\mathrm{pSi}$ for both kinds of passivation. Red and blue symbols represent $\mathrm{pSi}+\mathrm{F}$ and $\mathrm{pSi}+\mathrm{H}$, respectively.

where $E_{\mathrm{Mol}}, E_{\mathrm{X}+\mathrm{DB}}$, and $E_{\mathrm{X}+\mathrm{DB}+\mathrm{Mol}}$ are the energies of the molecule, the $\mathrm{X}$-passivated nanopore $(\mathrm{X}=\mathrm{H}$ or $\mathrm{F}$ ) with a $\mathrm{Si}$ dangling bond (DB) and the optimized complex formed by the X-passivated pSi with a gas molecule adsorbed on a DB, respectively. We have included the basis set superposition error correction $\left(E_{\mathrm{BSSE}}\right)$, given by the difference between the energies obtained with the individual and the combined basis-set functions of the pSi and the adsorbed molecule. ${ }^{62}$ A stronger adsorption of the molecule on the nanopore is indicated by a higher, or more positive, value of $E_{\mathrm{A}}$.

After comparing the results of the adsorption energies and bond distances, it can be seen that the numerical results for $\mathrm{H}$ and $\mathrm{F}$ passivation follow each other very closely, indicating that the chemical composition on the superficial layer does not affect in a significant way the adsorption strength of the molecules. The larger $E_{\mathrm{A}}$ among all the studied cases corresponds to the $\mathrm{NO}_{2}$ molecule. For $\mathrm{H}$ - and F-passivation schemes, these energies are $3.58 \mathrm{eV}$ and $3.29 \mathrm{eV}$, respectively, when the bonding occurs through the $\mathrm{O}$ atom; and 3.41 and $2.87 \mathrm{eV}$, respectively, when the $\mathrm{N}$ atom is bonded to the pSi surface. Also, this molecule has the smallest bond distance between its $\mathrm{O}$ atom and the Si surface atom, being $1.77 \AA$ for H-passivation and $1.69 \AA$ for F-passivation; which is close to reported experimental Si-O bond length of $1.63 \AA^{63,64}$ For the Si-N bond, we found almost the same distance for $\mathrm{pSi}+\mathrm{H}$ and $\mathrm{pSi}+\mathrm{F}$, being 1.93 and $1.92 \AA$, respectively. These distances are shorter than those found for $\mathrm{Si}-\mathrm{N}$ bonds of $2.27 \AA .{ }^{65}$ On the other hand, the lowest $E_{\mathrm{A}}$ corresponds to the $\mathrm{NO}(\mathrm{O})$ molecule on the $\mathrm{pSi}+\mathrm{H}$ and the $\mathrm{SO}_{2}(\mathrm{~S})$ molecule on the $\mathrm{pSi}+\mathrm{F}$, with $E_{\mathrm{A}}$ of $0.58 \mathrm{eV}$ and $0.19 \mathrm{eV}$, and bond distances of 2.45 and $2.49 \AA$, respectively.
These results seem to indicate that the $\mathrm{NO}_{2}$ and $\mathrm{SO}_{2}$ molecules, when interacting through their $\mathrm{O}$ atoms, having the strongest binding energy, have a strong chemical interaction with the surface of the $\mathrm{H}$ - or F-passivated pSi.

For a NO molecule adsorbed on pSi with $\mathrm{H}(\mathrm{F})$ passivation, the corresponding adsorption energies are $0.58 \mathrm{eV}(0.83 \mathrm{eV})$ and $1.71 \mathrm{eV}(1.57 \mathrm{eV})$ when the bonding occurs through $\mathrm{O}$ and $\mathrm{N}$, respectively. The NO-pSi distances are $2.45 \AA$ (2.61 $\AA$ ) when the bonding occurs through $\mathrm{O}$, and $1.93 \AA(2.07 \AA)$ when the bonding occurs through $\mathrm{N}$, exceeding the experimental reported results in both cases. For the $\mathrm{H}$-passivated case, the adsorption energy of $\mathrm{SO}_{2}(\mathrm{~S})(0.74 \mathrm{eV})$ is larger than that of the F-passivated case, but they have a similar bond distance of $2.44 \AA$. For $\mathrm{SO}_{2}(\mathrm{O})$, the adsorption energies and the bond distances are $1.50 \mathrm{eV}, 1.26 \mathrm{eV}$ and $1.76 \AA$, $1.72 \AA$, for the $\mathrm{H}$ - and F-passivated pSi, respectively. These results show that the $\mathrm{NO}_{2}$ has a tendency to get tightly bonded to the pSi surface, which hints that pSi may not be suitable for its detection since the adsorption of $\mathrm{NO}_{2}$ could lead to a fundamental change on the surface chemistry of the pSi which may not be able to be reversed.

For a better understanding, the adsorption reaction could be described as $\mathrm{pSi}_{(n-1)}+\mathrm{X} \rightarrow \mathrm{pSi}_{(n-1)} \mathrm{X}$, where pSi refers to $\mathrm{pSi}+\mathrm{H}$ or $\mathrm{pSi}+\mathrm{F}, n$ is the maximum number of $\mathrm{F}$ or $\mathrm{H}$ surface passivating atoms on the pSi surface and $(n-1)$ denotes the existence of at least one dandling bond formed during the pSi synthesis. $\mathrm{X}$ refers to the adsorbed molecule $\left(\mathrm{X}=\mathrm{NO}, \mathrm{NO}_{2}\right.$ or $\left.\mathrm{SO}_{2}\right)$ which can be adsorbed through both of its constituent atoms.

The van't Hoff-Arrhenius expression ${ }^{66,67}$ and the transition state theory give us the possibility to evaluate the recovery time of $\mathrm{pSi}+\mathrm{F}$ and $\mathrm{pSi}+\mathrm{H}$ after the molecule adsorption using the following equation: ${ }^{68,69}$

$$
t=A^{-1} \mathrm{e}^{\left(\frac{E_{\mathrm{A}}}{k_{\mathrm{B}} T}\right)}
$$

where $A$ denotes the frequency of oscillation of the adsorbed molecule. $E_{\mathrm{A}}$ is the adsorption energy; $k_{\mathrm{B}}$ is the Boltzmann's constant and $T$ is the test temperature. Notice that, at a specific temperature, a smaller value of $E_{\mathrm{A}}$ results in lower desorption time. Based on this, we compared the desorption time of the studied gas molecules adsorbed on $\mathrm{pSi}+\mathrm{F}$ and $\mathrm{pSi}+\mathrm{H}$. The $\mathrm{A}$ value was chosen to be $1012 \mathrm{~s}^{-1}$ as estimated in other studies. ${ }^{66-69}$ Desorption times were calculated at 273, 298 and $473 \mathrm{~K}$, see Fig. 5. As expected, desorption times depend on the adsorption energy of the molecule which in turn slightly depends on the pSi surface passivation. At the temperatures tested, the $\mathrm{NO}_{2}$ molecules will practically never desorb. All other results for desorption time are shown in Table 1. Both the adsorption energies and desorption time indicate that these structures could be suitable for detection of $\mathrm{NO}$ and $\mathrm{SO}_{2}$, while for $\mathrm{NO}_{2}$ the strong adsorption energy and high desorption time indicates that the molecule will not be able to desorb therefore making its detection less useful, however it could have potential for trapping these hazardous molecules. 


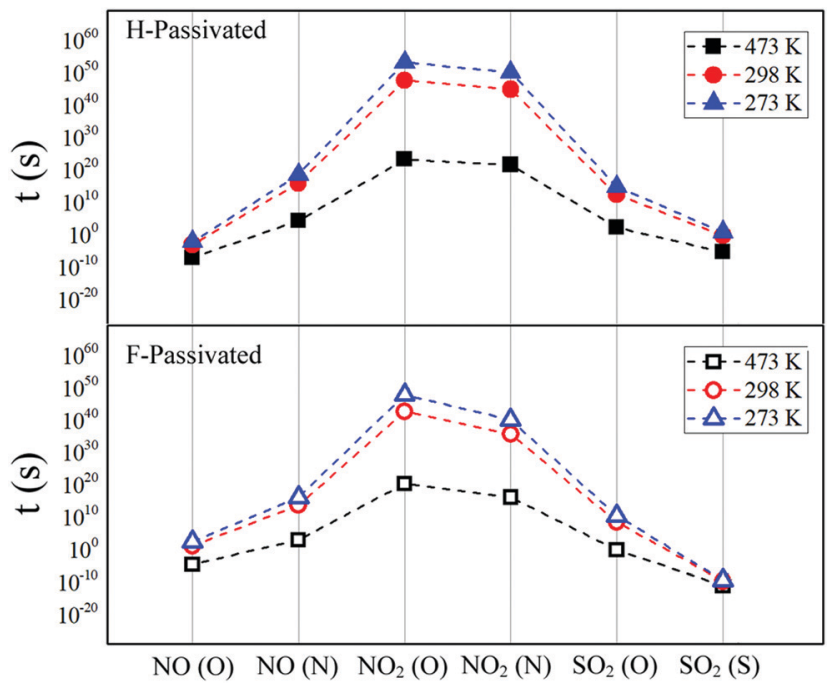

Fig. 5 Desorption times $(t)$ of molecules from $\mathrm{pSi}+\mathrm{H}$ (top panel, solid symbols) and pSi+F (bottom panel, open symbols). Triangle, circles and square symbols represent the different tested temperatures: 273, 298, and $473 \mathrm{~K}$, respectively.

\section{Relative stability and charge transfer}

The relative energetic stability of the nanostructures with an adsorbed molecule is approximated by the Gibbs free energy of formation $(\delta G):^{16}$

$$
\delta G=-E_{\mathrm{C}}+\sum \chi_{\mathrm{M}} \mu_{\mathrm{M}}
$$

where $E_{\mathrm{C}}$ and $\mu_{\mathrm{M}}$ are the cohesive energies per atom of the nanostructure and the chemical species $\mathrm{M}(\mathrm{M}=\mathrm{Si}, \mathrm{H}, \mathrm{F}, \mathrm{C}, \mathrm{N}, \mathrm{S}$, and $\mathrm{O}$ ) in their natural form, respectively, calculated with the same level of theory described above. $\chi_{M}$ is the molar fraction of atomic species $\mathrm{M}$, satisfying $\sum_{\mathrm{M}} \chi_{\mathrm{M}}=1 . E_{\mathrm{C}}$ can be calculated as:

$$
E_{\mathrm{C}}=\frac{\left(\sum_{\mathrm{M}} N_{\mathrm{M}} E_{\mathrm{M}}-E_{\mathrm{T}}\right)}{N_{\mathrm{T}}},
$$

where $E_{\mathrm{M}}$ and $E_{\mathrm{T}}$ are the total energies of an atom of type $\mathrm{M}$ and the complex formed by the adsorbed molecule and the nanopore, respectively. $N_{\mathrm{M}}$ and $N_{\mathrm{T}}$ are the number of atoms of species $\mathbf{M}$ and the total number of atoms in the system's supercell, respectively. More negative values of $\delta G$ indicate chemical stability and exothermic formation processes. The results

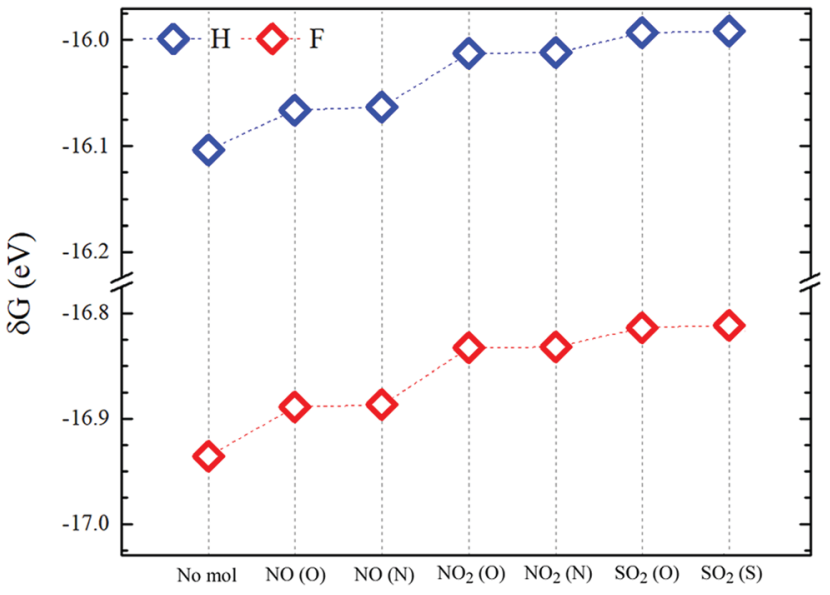

Fig. 6 Formation energies $(\delta G)$ of molecule-pSi complexes for fluorine (red symbols) and hydrogen (blue symbols) passivation. "No mol" denotes the passivated pSi without adsorbed molecules.

for all the molecules and configurations, plus the fully passivated case without molecules (called "No mol"), is showed in Fig. 6, along the hypothetical cases of adsorption on H-passivated pSi. Observe that both formation energies are negative, thus indicating that both structures are stable, which is expected due to the high number of $\mathrm{Si}$ atoms that conform the pSi framework; however, the fluorinated cases are substantially more stable, coinciding with the reported results that fluorination diminishes the reactivity of Si surfaces in comparison to hydrogen passivation. Although the inclusion of the molecules on the pore surface rises the formation energy, these changes are not drastic, hence the adsorption is possible. The difference between $\delta G$ for both passivation schemes is consistent around $0.85 \mathrm{eV}$, and the following trend is observed for $\delta G$ : $\mathrm{No} \mathrm{mol}<\mathrm{NO}<\mathrm{NO}_{2}<\mathrm{SO}_{2}$, for both passivation schemes, regardless of the atom of the molecule bonded to the pSi.

In this way, to better understand the interaction between $\mathrm{pSi}$ and the toxic molecules, we calculated the molecule charge excess by using the Voronoi population analysis. Fig. 7 shows the charge excess on the adsorbed molecules, where positive values indicate that the molecule transferred electrons (negative charge) to the passivated pSi. This is the case of NO in its two adsorption configurations on the $\mathrm{pSi}+\mathrm{F}$. Likewise, negative values of the charge transfer indicate an electronic charge transfer from the pSi to the molecule, as occurs for the rest

\begin{tabular}{|c|c|c|c|c|c|c|}
\hline & \multicolumn{6}{|c|}{ Test temperature } \\
\hline & \multicolumn{2}{|l|}{$273 \mathrm{~K}$} & \multicolumn{2}{|l|}{$298 \mathrm{~K}$} & \multicolumn{2}{|l|}{$473 \mathrm{~K}$} \\
\hline & $\mathrm{pSi}+\mathrm{H}$ & $\mathrm{pSi}+\mathrm{F}$ & $\mathrm{pSi}+\mathrm{H}$ & $\mathrm{pSi}+\mathrm{F}$ & $\mathrm{pSi}+\mathrm{H}$ & $\mathrm{pSi}+\mathrm{F}$ \\
\hline NO (O) & $2.22 \times 10^{2}$ & $5.70 \times 10^{-3}$ & $1.15 \times 10^{1}$ & $7.14 \times 10^{-4}$ & $2.59 \times 10^{-5}$ & $7.88 \times 10^{-8}$ \\
\hline NO $(\mathrm{N})$ & $1.17 \times 10^{16}$ & $3.84 \times 10^{18}$ & $4.27 \times 10^{13}$ & $8.61 \times 10^{15}$ & $8.62 \times 10^{2}$ & $2.06 \times 10^{4}$ \\
\hline $\mathrm{NO}_{2}(\mathrm{O})$ & $5.38 \times 10^{47}$ & $1.19 \times 10^{53}$ & $4.33 \times 10^{42}$ & $3.42 \times 10^{47}$ & $1.96 \times 10^{20}$ & $1.67 \times 10^{23}$ \\
\hline $\mathrm{NO}_{2}(\mathrm{~N})$ & $1.12 \times 10^{40}$ & $1.09 \times 10^{50}$ & $3.98 \times 10^{35}$ & $5.59 \times 10^{44}$ & $1.21 \times 10^{16}$ & $3.60 \times 10^{21}$ \\
\hline $\mathrm{SO}_{2}(\mathrm{O})$ & $2.68 \times 10^{10}$ & $5.85 \times 10^{14}$ & $2.90 \times 10^{8}$ & $2.74 \times 10^{12}$ & $6.96 \times 10^{-1}$ & $1.67 \times 10^{2}$ \\
\hline $\mathrm{SO}_{2}(\mathrm{~S})$ & $3.52 \times 10^{-10}$ & $6.45 \times 10^{0}$ & $1.78 \times 10^{-10}$ & $4.48 \times 10^{-1}$ & $8.80 \times 10^{-12}$ & $3.72 \times 10^{-6}$ \\
\hline
\end{tabular}

Table 1 Molecule desorption time in seconds 


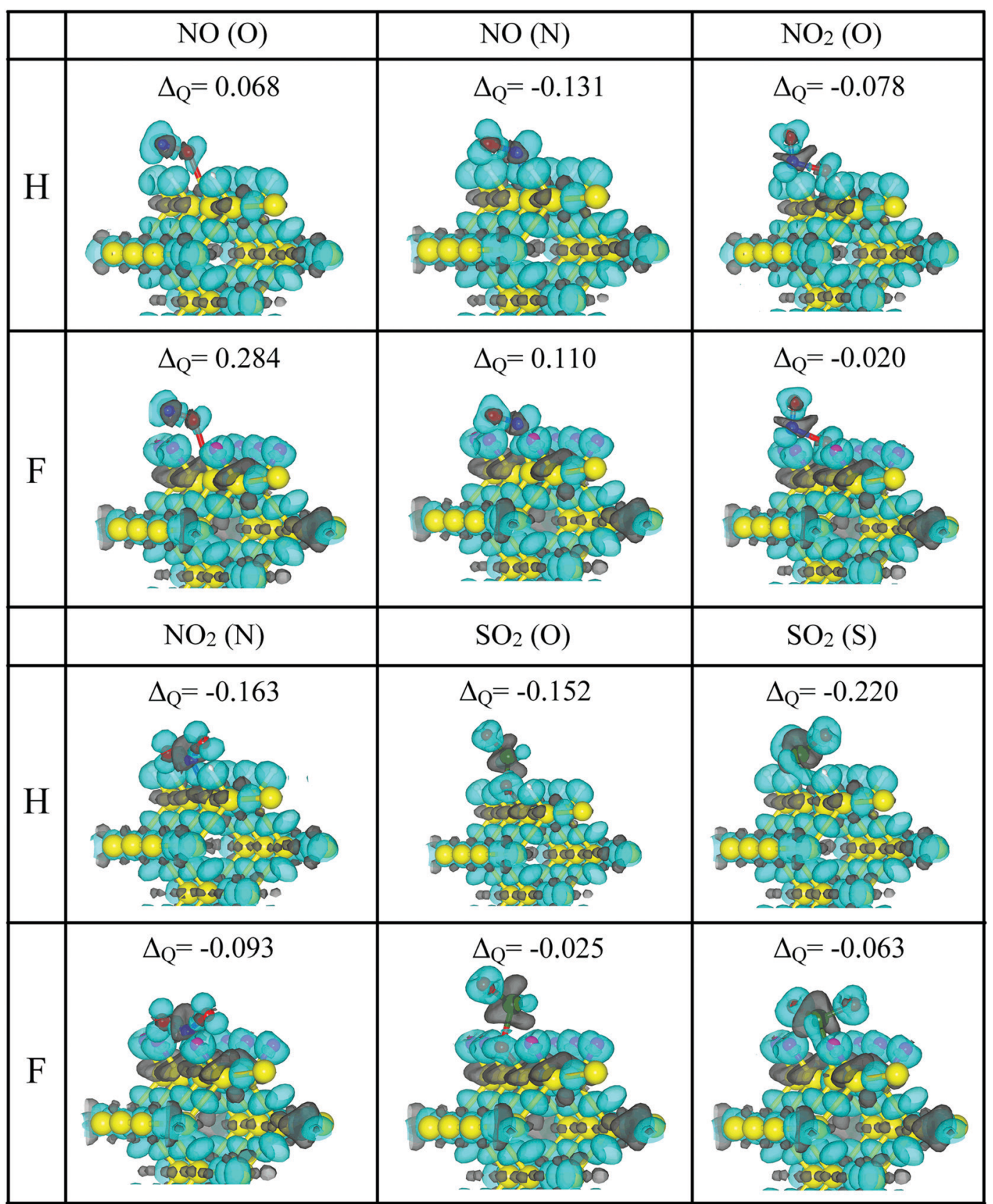

Fig. 7 Molecule charge excess $(\Delta Q)$ and $\mathrm{CDD}$ isosurface plots of $\mathrm{NO}, \mathrm{NO}_{2}$ and $\mathrm{SO}_{2}$ molecules adsorbed on $\mathrm{F}$ - (magenta) and $\mathrm{H}$-passivated (blue) pSi. The $\mathrm{pSi}+\mathrm{F}$ and $\mathrm{pSi}+\mathrm{H}$ cases are indicated in the left column as $\mathrm{F}$ and $\mathrm{H}$, respectively. The isosurfaces have a value of 0.06 electron per $\AA^{3}$. Cyan (gray) isosurfaces denote gain (loss) of electronic charge in comparison with the ground-state charge density of the isolated atoms, for $\mathrm{H}$ or F-passivated pSi systems.

of the cases. These results are complemented by the threedimensional isosurface plots of CDD (eqn (1)). In the cases of $\mathrm{NO}_{2}$ and $\mathrm{SO}_{2}$, there are small grey lobes around the Si dangling bond, which indicates that $\mathrm{pSi}$ transfers electronic charge to the molecules. On the other hand, these small gray lobes are not observed on the NO cases, where the molecule is losing electronic charge. Also, when $\mathrm{NO}_{2}$ is bonded through an $\mathrm{O}$ atom, there is a gain of charge density around the $\mathrm{Si}-\mathrm{O}$ bond, and an extended loss of charge between that $\mathrm{O}$ atom and the $\mathrm{N}$ one, for both passivation cases, indicating the formation of a
Si-O covalent bond, confirmed by the large magnitude of the adsorption energy found in this case (see Fig. 4). Changes of the former electrical resistivity of pSi after the adsorption of toxic molecules are expected due to these charge transfers, which could be measured by experiments. ${ }^{7}$

\section{Electronic properties}

Fig. 8 shows the electronic band structures and the partial densities of the with the adsorbed molecules. Upon NO adsorption, two impurity levels, one above and the other below the 

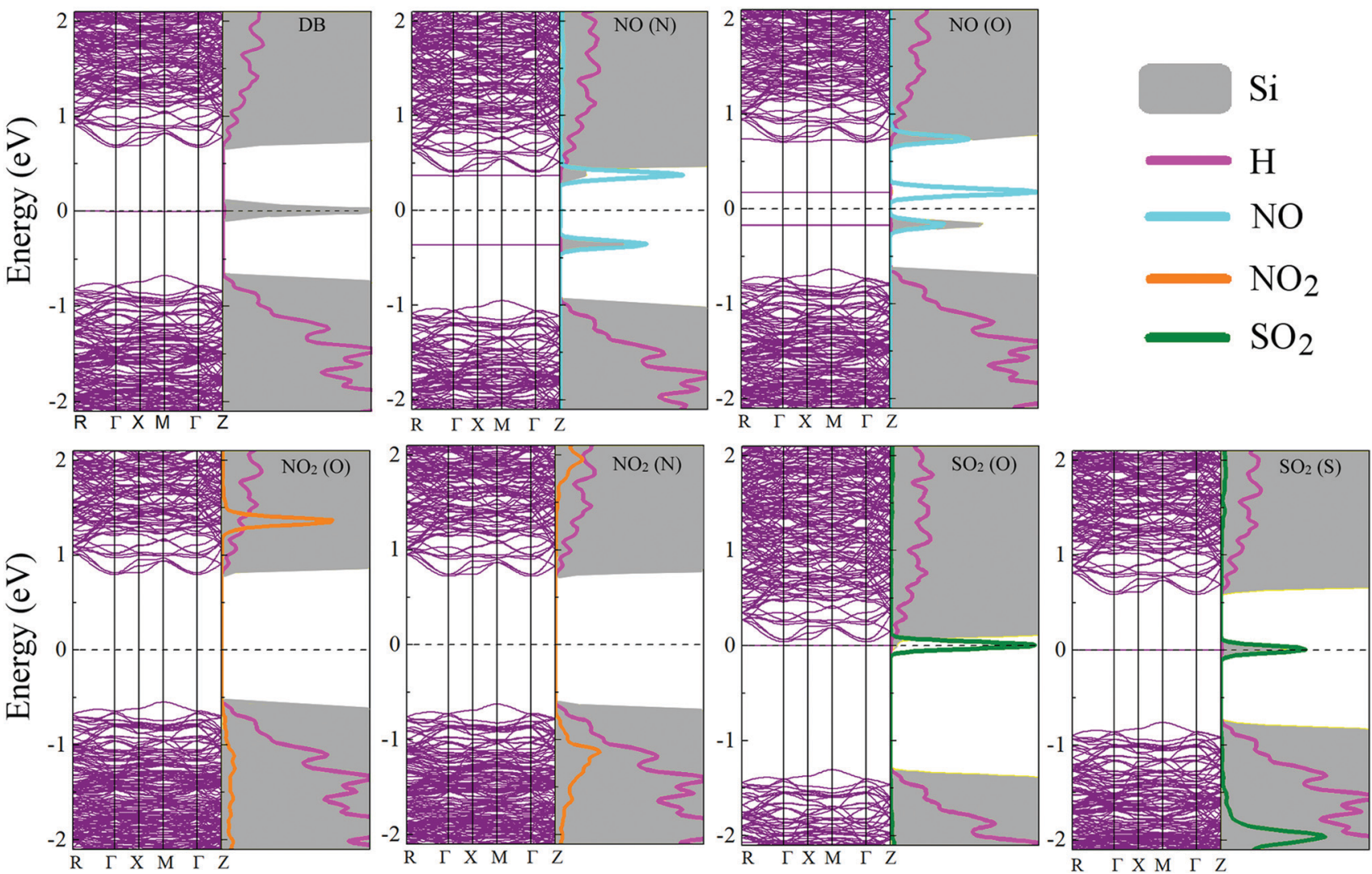

Fig. 8 Electronic band structure and partial density of states for the studied molecule-pSi+F systems. Fermi level is indicated with a dashed line at 0 eV.

Fermi level, appear with energy differences that lead to band gaps of $0.340 \mathrm{eV}$ for $\mathrm{NO}(\mathrm{O})$ and $1.344 \mathrm{eV}$ for $\mathrm{NO}(\mathrm{N})$. These flat states suggest that there are highly localized orbitals around the molecule. On the adsorption of $\mathrm{NO}_{2}$, no impurity states appear within the band gap, but there is a contribution from the molecule to the electronic states inside the conduction and the valence bands, this could be understood in terms of the stronger adsorption of the $\mathrm{NO}_{2}$ onto the pSi surface, which leads to less localized states compared to the other molecules that have weaker bonds to the pore, i.e., lower adsorption energies, and in consequence, quicker desorption times than the $\mathrm{NO}_{2}$ cases (see Fig. 5). In this case, the value of the band gap is equal to that of the fully fluorinated case $(1.47 \mathrm{eV})$ indicating that a strong bond is formed between the $\mathrm{Si}$ and the constituent atoms of the molecule, which is similar to the $\mathrm{F}$ passivation leading to a band gap without defect states. For both $\mathrm{SO}_{2}$ adsorption configurations, a localized state emerges within the former $\mathrm{pSi}+\mathrm{F}$ band gap, thus modifying its value. These modified band gaps are $0.037 \mathrm{eV}$ for $\mathrm{SO}_{2}(\mathrm{O})$ and $0.585 \mathrm{eV}$ for $\mathrm{SO}_{2}(\mathrm{~S})$. In the case of $\mathrm{SO}_{2}(\mathrm{O})$ adsorbed on $\mathrm{pSi}+\mathrm{F}$, the Fermi level shifts towards the conduction band, similar to a n-type doping effect, which could facilitate the electronic detection of this molecule.

Fig. 9 shows the isosurface plots of the eigenfunctions of the valence band maximum (HOMO) and conduction band minimum (LUMO) energy eigenvalues, together with the magnitudes of the bandgaps of the studied systems. For both NO adsorption cases showed in Fig. 9, observe that the HOMO and LUMO isosurfaces are concentrated around the molecule, which corroborates that the flat states showed in Fig. 8 come mostly from the NO molecule. On the other hand, the HOMO and LUMO isosurfaces for the $\mathrm{NO}_{2}$ adsorption cases indicate that the molecule does not contribute to the corresponding electronic states since these isosurfaces are concentrated around the $\mathrm{Si}-\mathrm{Si}$ bonds, in agreement with the partial density of states analysis (Fig. 8). Finally, the isosurfaces corresponding to both $\mathrm{SO}_{2}$ adsorption cases show that the HOMOs are concentrated around the molecule, and the LUMOs around the $\mathrm{Si}$ atoms, respectively indicating that the HOMO flat states come from the molecule, and the LUMO states come mostly from the Si atoms. Summarizing, for the cases where impurity states are found within the former $\mathrm{pSi}$ band gap, HOMO and LUMO isosurface plots confirm that these flat states come mostly from the adsorbed molecules, in agreement with Fig. 8. Some of the studied cases could be interesting for the design of optical sensors, where the pSi optical band gap change, upon toxic molecule adsorption, could be detected through the corresponding change in the wavelength of the adsorbed radiation.

\section{Conclusions}

The adsorption of the hazardous gas molecules $\mathrm{NO}, \mathrm{NO}_{2}$ and $\mathrm{SO}_{2}$, on fluorine-passivated porous silicon has been theoretically 


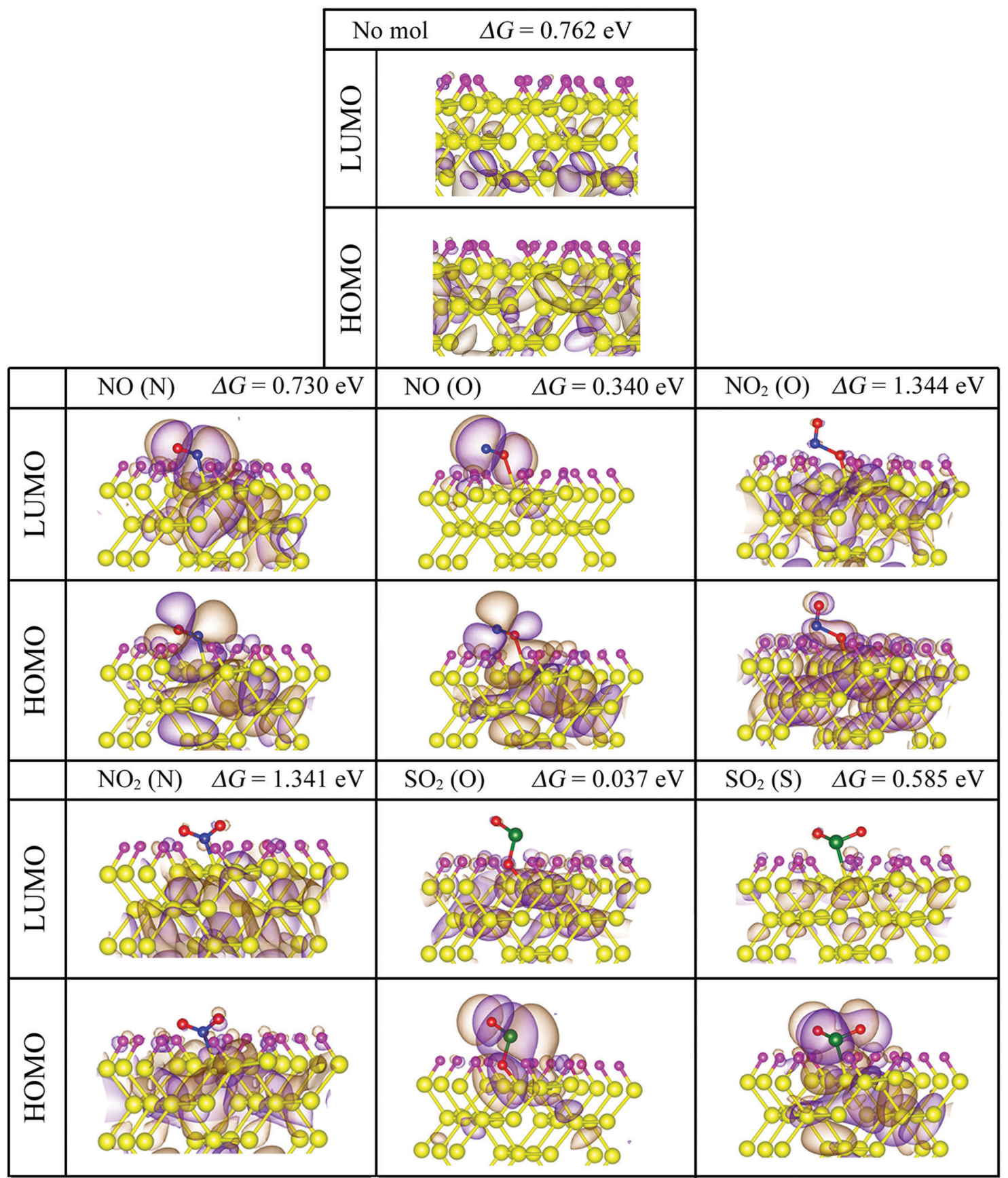

Fig. 9 Isosurfaces of HOMO and LUMO orbitals, with corresponding band gaps for all studied molecules adsorbed on F-passivated pSi. The isosurfaces have a value of 0.03 electron per $\AA^{3}$. Purple (brown) isosurfaces represent positive (negative) values of the plotted wavefunction on the 3D grid.

studied and compared with the hypothetical case of adsorption on hydrogen-passivated porous silicon.

We found that the molecule adsorption energies, between the toxic molecules and the $\mathrm{pSi}+\mathrm{F}$, depend on the molecule which is adsorbed on the pSi and these adsorption energies are numerically very close to the ones obtained for $\mathrm{pSi}+\mathrm{H}$, which implies that these values barely depend on the pSi passivation. In most cases, the band gap diminishes after the molecule adsorption, which can modify the conductivity of pSi facilitating the detection of different kinds of gas molecules. Especially for
$\mathrm{SO}_{2}(\mathrm{O})$ case, where there is a shift of the Fermi level to the conduction band, similar to a n-type doping effect.

The $\mathrm{NO}_{2}$ molecule has the largest adsorption energy on $\mathrm{pSi}$, however, it does not significantly modify the former bandgap of the passivated pSi. Due to the large adsorption energies found for $\mathrm{NO}_{2}, \mathrm{pSi}+\mathrm{F}$ could be used as a chemical trap for this hazardous gas. Moreover, the results suggest that $\mathrm{pSi}+\mathrm{F}$ is suitable for NO detection since the adsorption energies are slightly above $0.5 \mathrm{eV}$, which indicates that a weak chemical bond is formed, and there is also a marked reduction of the 
former bandgap of $\mathrm{pSi}+\mathrm{F}$. Besides, it has the best relative stability. Also, some of these cases could be interesting for the design of optical sensors, since the optical band gap of pSi changes upon toxic molecule adsorption.

This work presents theoretical evidence of the potential applications of fluorinated pSi as chemical traps or sensors of different types of hazardous gases. We believe that this study can help to the development of future experimental and theoretical research in the fields of electronic engineering and environmental protection.

\section{Conflicts of interest}

There are no conflicts to declare.

\section{Acknowledgements}

This work was partially supported through the multidisciplinary projects IPN-SIP 2020-2093, -2091, -2106, and UNAM-PAPIIT IN109320. Computations were done at supercomputer Miztli of DGTIC-UNAM (projects LANCAD-UNAM-DGTIC-180 and -381). J. E. Santana and F. de Santiago are grateful for the graduate scholarship granted by CONACYT and BEIFI.

\section{Notes and references}

1 Health Effects Institute, State of Global Air 2019, www. stateofglobalair.org/report, 2019, accessed 21 Oct 2020.

2 R. Kumar, O. Al-Dossary, G. Kumar and A. Umar, Zinc oxide nanostructures for $\mathrm{NO}_{2}$ gas-sensor applications: A review, Nano-Micro Lett., 2015, 7(2), 97-120.

3 R. K. Srivastava Controlling $\mathrm{SO}_{2}$ emissions - a review of technologies, United States Environmental Protection Agency, Office of Research and Development, 2000, pp. 397-422.

4 S. T. Shishiyanu, T. S. Shishiyanu and O. I. Lupan, Sensing characteristics of tin-doped $\mathrm{ZnO}$ thin films as $\mathrm{NO}_{2}$ gas sensor, Sens. Actuators, B, 2005, 107(1), 379-386.

5 C. Baratto, et al., A novel porous silicon sensor for detection of sub-ppm $\mathrm{NO}_{2}$ concentrations, Sens. Actuators, B, 2001, 77(1-2), 62-66.

6 S. G. Chatterjee, et al., Graphene-metal oxide nanohybrids for toxic gas sensor: A review, Sens. Actuators, B, 2015, 221, 1170-1181.

7 K. Xu, N. Liao, W. Xue and H. Zhou, Predicting gases sensing performance of $\alpha-\mathrm{MoO}_{3}$ from nano-structural and electronic properties, Appl. Surf. Sci., 2020, 509, 144913.

8 D. Kwak, M. Wang, K. J. Koski, L. Zhang, H. Sokol, R. Maric and Y. Lei, Molybdenum trioxide $\left(\alpha-\mathrm{MoO}_{3}\right)$ nanoribbons for ultrasensitive ammonia $\left(\mathrm{NH}_{3}\right)$ gas detection: Integrated experimental and density functional theory simulation studies, ACS Appl. Mater. Interfaces, 2019, 11(11), 10697-10706.

9 N. Liao, H. Zhou, B. Zheng and W. Xue, Silicon oxycarbidederived carbon as potential $\mathrm{NO}_{2}$ gas sensor: a first principles' study, IEEE Electron Device Lett., 2018, 39(11), 1760-1763.
10 G. K. Walia and D. K. K. Randhawa, Adsorption and dissociation of sulfur-based toxic gas molecules on silicene nanoribbons: A quest for high-performance gas sensors and catalysts, J. Mol. Model., 2018, 24(4), 94.

11 A. Sahithi and K. Sumithra, Adsorption and sensing of CO and $\mathrm{NH}_{3}$ on chemically modified graphene surfaces, $R S C$ Adv., 2020, 10(69), 42318-42326.

12 W. Wang, Y. Zhang, C. Shen and Y. Chai, Adsorption of CO molecules on doped graphene: A first-principles study, AIP Adv., 2016, 6(2), 025317.

13 C. Yi, W. Wang and C. Shen, The adsorption properties of CO molecules on single-layer graphene nanoribbons, AIP Adv., 2014, 4(3), 031330.

14 A. Miranda, F. de Santiago, L. A. Pérez and M. Cruz-Irisson, Silicon nanowires as potential gas sensors: A density functional study, Sens. Actuators, B, 2017, 242, 1246-1250.

15 F. De Santiago, A. Trejo, A. Miranda, F. Salazar, E. Carvajal, L. A. Pérez and M. Cruz-Irisson, Carbon monoxide sensing properties of B-, Al-and Ga-doped Si nanowires, Nanotechnology, 2018, 29(20), 204001.

16 F. de Santiago, et al., Quasi-one-dimensional silicon nanostructures for gas molecule adsorption: A DFT investigation, Appl. Surf. Sci., 2019, 475, 278-284.

17 V. Lehmann and U. Gösele, Porous silicon formation: A quantum wire effect, Appl. Phys. Lett., 1991, 58(8), 856-858.

18 K. Q. Peng, et al., Synthesis of large-area silicon nanowire arrays via self-assembling nanoelectrochemistry, Adv. Mater., 2002, 14(16), 1164-1167.

19 F. Bai, et al., One-step synthesis of lightly doped porous silicon nanowires in $\mathrm{HF} / \mathrm{AgNO}_{3} / \mathrm{H}_{2} \mathrm{O}_{2}$ solution at room temperature, J. Solid-State Chem., 2012, 196, 596-600.

20 G. Bomchil, R. Herino, K. Barla and J. C. Pfister, Pore size distribution in porous silicon studied by adsorption isotherms, J. Electrochem. Soc., 1983, 130(7), 1611.

21 C. C. Cheng and J. T. Yates Jr, H-Induced surface restructuring on $\mathrm{Si}(100)$ : Formation of higher hydrides, Phys. Rev. B: Condens. Matter Mater. Phys., 1991, 43(5), 4041.

22 Z. H. Lu, K. Griffiths, P. R. Norton and T. K. Sham, Adsorption of atomic hydrogen on $\mathrm{Si}(100)$ surface, Phys. Rev. Lett., 1992, 68(9), 1343.

23 L. Seals, J. L. Gole, L. A. Tse and P. J. Hesketh, Rapid, reversible, sensitive porous silicon gas sensor, J. Appl. Phys., 2002, 91(4), 2519-2523.

24 F. A. Harraz, Porous silicon chemical sensors and biosensors: A review, Sens. Actuators, B, 2014, 202, 897-912.

25 A. M. Rossi, et al., Porous silicon biosensor for detection of viruses, Biosens. Bioelectron., 2007, 23(5), 741-745.

26 G. Rong, et al., Nanoscale porous silicon waveguide for label-free DNA sensing, Biosens. Bioelectron., 2008, 23(10), 1572-1576.

27 I. Schechter, M. Ben-Chorin and A. Kux, Gas sensing properties of porous silicon, Anal. Chem., 1995, 67(20), 3727-3732.

28 S. E. Lewis, J. R. DeBoer, J. L. Gole and P. J. Hesketh, Sensitive, selective, and analytical improvements to a porous silicon gas sensor, Sens. Actuators, B, 2005, 110(1), 54-65. 
29 C. Baratto, E. Comini, G. Faglia, G. Sberveglieri, G. Di Francia, F. De Filippo and L. Lancellotti, Gas detection with a porous silicon-based sensor, Sens. Actuators, B, 2000, 65(13), 257-259.

30 N. Naderi, M. R. Hashim and T. S. T. Amran, Enhanced physical properties of porous silicon for improved hydrogen gas sensing, Superlattices Microstruct., 2012, 51(5), 626-634.

31 A. Trejo, M. Calvino and M. Cruz-Irisson, Chemical surface passivation of 3C-SiC nanocrystals: A first-principle study, Int. J. Quantum Chem., 2010, 110(13), 2455-2461.

32 R. Rurali, Colloquium: Structural, electronic, and transport properties of silicon nanowires, Rev. Mod. Phys., 2010, 82(1), 427.

33 M. Amato and R. Rurali, Surface physics of semiconducting nanowires, Prog. Surf. Sci., 2016, 91(1), 1-28.

34 R. Singh, Effect of hydrogen on ground state properties of silicon clusters (SinHm; $n=11-15, m=0-4$ ): a density functional based tight binding study, J. Phys.: Condens. Matter, 2008, 20(4), 045226.

$35 \mathrm{~L}$. Lin, et al., The role of surface terminations on the band structure and optical properties of silicon nanonets, Appl. Surf. Sci., 2011, 257(6), 2032-2037.

36 S. Louhibi, et al., The effects of hydrogen on the electronic properties of silicon, Superlattices Microstruct., 2005, 37(2), 115-126.

37 A. Manilov and V. Skryshevsky, Hydrogen in porous silicon A review, Mater. Sci. Eng., B, 2013, 178(15), 942-955.

38 E. Ramos, et al., Theoretical study of the electronic properties of silicon nanocrystals partially passivated with $\mathrm{Cl}$ and F, J. Phys. Chem. C, 2012, 116(6), 3988-3994.

39 Y. Ma, X. Pi and D. Yang, Fluorine-passivated silicon nanocrystals: surface chemistry versus quantum confinement, J. Phys. Chem. C, 2012, 116(9), 5401-5406.

40 A. Trejo, et al., Ab-initio study of anisotropic and chemical surface modifications of $\beta$-SiC nanowires, J. Mol. Model., 2013, 19(5), 2043-2048.

41 Y. Ma, et al., Theoretical study of chlorine for silicon nanocrystals, J. Phys. Chem. C, 2011, 115(26), 12822-12825.

42 A. Martínez, et al., Electronic structure of silicon nanocrystals passivated with nitrogen and chlorine, J. Phys. Chem. C, 2010, 114(29), 12427-12431.

43 T. A. Miura, M. Niwano, D. Shoji and N. Miyamoto, Kinetics of oxidation on hydrogen-terminated $\mathrm{Si}(100)$ and (111) surfaces stored in air, J. Appl. Phys., 1996, 79(8), 4373-4380.

44 F. Ptashchenko, Formation of acceptor states on the silicon hydroxylated surface upon $\mathrm{NO}_{2}$ molecules adsorption, Phys. Status Solidi B, 2018, 255(3), 1700499.

45 M. Li, M. Hu, W. Yan, S. Ma, P. Zeng and Y. Qin, $\mathrm{NO}_{2}$ sensing performance of p-type intermediate size porous silicon by a galvanostatic electrochemical etching method, Electrochim. Acta, 2013, 113, 354-360.

46 T. Sunada, T. Yasaka, M. Takakura, T. Sugiyama, S. Miyazaki and $\mathbf{M}$. Hirose, The role of fluorine termination in the chemical stability of HF-treated Si surfaces, Jpn. J. Appl. Phys., 1990, 29(12A), L2408.

47 C. J. Wu and E. A. Carter, Mechanistic predictions for fluorine etching of silicon(100), J. Am. Chem. Soc., 1991, 113(24), 9061-9062.

48 T. Hoshino and Y. Nishioka, Theoretical estimation of the energy differences among $\mathrm{OH}-, \mathrm{F}-$, and $\mathrm{H}$-terminations of the Si surface, Jpn. J. Appl. Phys., 1999, 38(12R), 6855.

49 T. Hoshino and Y. Nishioka, Etching process of SiO2 by $\mathrm{HF}$ molecules, J. Chem. Phys., 1999, 111(5), 2109-2114.

50 T. Chowdhury, R. Hidayat, T. R. Mayangsari, J. Gu, H. L. Kim, J. Jung and W. J. Lee, Density functional theory study on the fluorination reactions of silicon and silicon dioxide surfaces using different fluorine-containing molecules, J. Vac. Sci. Technol., A, 2019, 37(2), 021001.

51 W. Kohn and L. J. Sham, Self-consistent equations including exchange and correlation effects, Phys. Rev., 1965, 140(4A), A1133.

52 P. Hohenberg and W. Kohn, Inhomogeneous electron gas, Phys. Rev., 1964, 136(3B), B864.

53 J. P. Perdew, K. Burke and M. Ernzerhof, Generalized gradient approximation made simple, Phys. Rev. Lett., 1996, 77(18), 3865.

54 E. Artacho, et al., Linear-scaling ab-initio calculations for large and complex systems, Phys. Status Solidi B, 1999, 215(1), 809-817.

$55 \mathrm{~J}$. Junquera, et al., Numerical atomic orbitals for linearscaling calculations, Phys. Rev. B: Condens. Matter Mater. Phys., 2001, 64(23), 235111.

56 N. Troullier and J. L. Martins, Efficient pseudopotentials for plane-wave calculations, Phys. Rev. B: Condens. Matter Mater. Phys., 1991, 43(3), 1993.

57 L. Kleinman and D. Bylander, Efficacious form for model pseudopotentials, Phys. Rev. Lett., 1982, 48(20), 1425.

58 J. M. Soler, et al., The SIESTA method for ab initio order-N materials simulation, J. Phys.: Condens. Matter, 2002, 14(11), 2745.

59 H. J. Monkhorst and J. D. Pack, Special points for Brillouinzone integrations, Phys. Rev. B: Condens. Matter Mater. Phys., 1976, 13(12), 5188.

60 C. Fonseca Guerra, et al., Voronoi deformation density (VDD) charges: Assessment of the Mulliken, Bader, Hirshfeld, Weinhold, and VDD methods for charge analysis, J. Comput. Chem., 2004, 25(2), 189-210.

61 K. Oura, M. Katayama, A. V. Zotov, V. G. Lifshits and A. A. Saranin, Surface Science, Springer Berlin Heidelberg, Berlin, Heidelberg, 2003.

62 S. F. Boys and F.d. Bernardi, The calculation of small molecular interactions by the differences of separate total energies. Some procedures with reduced errors, Mol. Phys., 1970, 19(4), 553-566.

63 J. Koput, An ab initio study on the potential energy surface of large-amplitude motions for disiloxane, J. Phys. Chem., 1995, 99(43), 15874-15880.

64 F. Weinhold and R. West, The nature of the silicon-oxygen bond, Organometallics, 2011, 30(21), 5815-5824. 
65 M. S. Gordon, et al., Nature of the silicon-nitrogen bond in silatranes, Organometallics, 1991, 10(8), 2657-2660.

$66 \mathrm{~S}$. Peng, et al., Ab initio study of $\mathrm{CNT} \mathrm{NO}_{2}$ gas sensor, Chem. Phys. Lett., 2004, 387(4-6), 271-276.

67 Y.-H. Zhang, et al., Improving gas sensing properties of graphene by introducing dopants and defects: a firstprinciples study, Nanotechnology, 2009, 20(18), 185504.
68 D. Chen, et al., Pristine and $\mathrm{Cu}$ decorated hexagonal InN monolayer, a promising candidate to detect and scavenge $\mathrm{SF}_{6}$ decompositions based on first-principle study, J. Hazard. Mater., 2019, 363, 346-357.

69 K. Patel, et al., A new flatland buddy as toxic gas scavenger: A first principles study, J. Hazard. Mater., 2018, 351, 337-345. 